\title{
Influence of carbon or nitrogen dopants on the electronic structure, optical properties and photocatalytic activity of partially reduced titanium dioxide
}

\author{
V.P. Zhukov ${ }^{1}$, M. G. Kostenko ${ }^{1}$, A. A. Rempel ${ }^{2}$, I. R. Shein ${ }^{1}$ \\ ${ }^{1}$ Institute of Solid State Chemistry, Ural Branch of the Russian Academy of Sciences, Russia \\ ${ }^{2}$ Institute of Metallurgy, Ural Branch of the Russian Academy of Sciences, Russia
}

Zhukov@ihim.uran.ru

PACS 71.20.Nr, 61.72.Ji, 71.55.i

DOI 10.17586/2220-8054-2019-10-3-374-382

\begin{abstract}
For titanium dioxide with anatase structure doped with carbon or nitrogen, the first-principle method of projector augmented waves (PAW) is used to calculate electronic band structure, to evaluate vacancy formation energy for the oxygen sublattice, and to analyze optical absorption. It is demonstrated that the presence of carbon dopants results in the stabilization of oxygen vacancies and leads to increased absorption in the visible spectrum, which can facilitate the photocatalytic activity. The presence of nitrogen dopant also facilitates vacancy stabilization but no increase in the interband absorption is expected in the visible spectrum, i.e., the presence of nitrogen dopant cannot be considered as a factor contributing to increased photocatalytic activity. It follows from the calculated data that the maximum photocatalytic activity should be expected for the partiallyreduced anatase doped with carbon because of the absorption in the visible spectrum that combines with long time of electron-hole recombination.
\end{abstract}

Keywords: titanium dioxide, vacancies, doping, electronic structure, optical absorption, photocatalysis.

Received: 12 February 2019

Revised: 22 April 2019

\section{Introduction}

Titanium dioxide with anatase structure is stable, inexpensive, and exhibits photocatalytic activity in the UV range, which makes it a promising material for application in the development of photocatalysts capable of operating efficiently under solar radiation [1-7]. To this end, the material has to be modified to enhance its optical absorption in the visible spectrum comprising a majority of the radiation energy, i.e., it is required to increase the number of photocatalytically active electron-hole pairs. At the same time, electrons and holes are required to retain high mobility, while their recombination rate should be low.

Doping with $2 p-, 3 p-, 6 p$ - and $3 d$ - elements is regarded to be an effective method for enhancing absorption in the visible spectrum and partial reduction of titanium dioxide. The reduction results in vacancies formed in the oxygen sublattice, which is also considered to be of utility for enhancing photocatalytic activity. In particular, a process of photocatalytic decomposition of organic molecules on partially-reduced anatase under visible light irradiation is reported in [8-12]. State-of-the-art theoretical methods developed for calculating electronic structure and properties can be instrumental in designing efficient photocatalysts. Such methods are useful for obtaining information about the states of oxygen vacancies and other defects present in the band gap. They can be applied to explore defect-related characteristics of optical absorption, to study mobility of current carriers and to determine electron-hole recombination rates. A significant amount of research has been reported in the literature, along with calculations of electronic band structure, calculations of defect formation energy [13-19], crystalline geometry modifications near dopants [13, 14, 16,18], and interpretation of optical [14,20], photoemission [14] and EPR spectra [14]. However, the cited references have some drawbacks that hinder realization of the state-of-the-art capabilities developed for modeling the electronic structure and characteristics of solids. Thus, the cited sources [13-17, 19,21] fail to include calculations of optical properties and to consider correlations between the band structure and the optical properties. Also disputable is the method applied in $[15-17,19,21]$ for calculating the formation energy of vacancies with the charge $q=+1$ or +2 . When calculating the formation energy of charged vacancies using this approach, the fault is that the presence of charge is modeled by introducing only a uniform charge distribution. However, in real objects, dopant atoms of carbon, nitrogen and other elements may be electron acceptors, which may cause a substantial effect on the chemical bonding around vacancies.

The goal of the present research is to provide the results of a new theoretical study exploring the issues related to the effect of vacancies on the photocatalytic activity of anatase. The study comprises calculations of band structure, reviews the energy aspects of vacancy formation, and provides assessment of optical absorption coefficients at various energies. In addition, an effort is made to model the electronic structure of charged vacancies by introducing impurity centers of nitrogen atoms, which transform vacancies into a charged state of $q=+1$, or carbon atoms, which result in 
a charged state of $q=+2$. The study allowed a number of conclusions to be made about the prospects for enhancing the photocatalytic performance of partially reduced anatase.

\section{Method for calculating electronic structure and vacancy formation energy}

A supercell method was applied to model the defect structure comprising structural vacancies and impurity atoms in titanium dioxide [22]. The extended cell was produced by twofold translations of tetragonal elementary cell of anatase (Fig. 1) in every crystallographic direction; and it contained 32 sites in the titanium sublattice and 64 sites in the oxygen sublattice. The supercell of the specified dimension allowed a minimum concentration of vacancies at $1.56 \%$ to be produced in the oxygen sublattice by removing one atom from the initially complete cell [23]. The calculations were carried out using a pseudo-potential projector augmented waves method (PAW) within the basis of plane waves realized in the VASP software package [24]. Software-provided PAW pseudo-potentials were used; and for the exchange-correlation potential the GGA approximation was applied. The calculations were carried out for the lattice composed of 27 vectors in the irreducible part of the Brillouin zone and with the maximum energy of plane waves at $350 \mathrm{eV}$. In all calculations, the accuracy of self-consistent total energy was no worse than $10^{-6} \mathrm{eV}$.

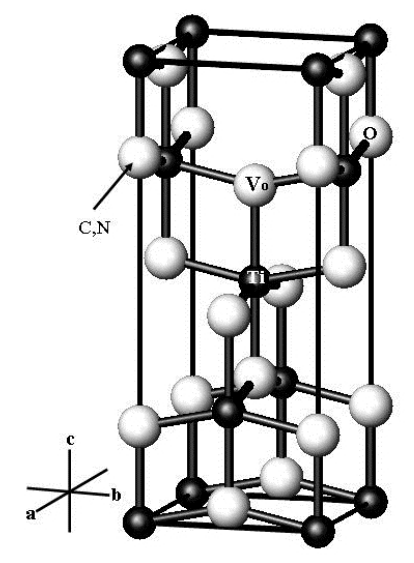

FIG. 1. A tetragonal structure of anatase. Titanium atoms are designated with the black balls, and oxygen atoms are designated with the light balls. Also shown are the oxygen vacancy sites VO and the sites of substituting carbon or nitrogen atoms

In all cases considered below, the lattice parameters and the atomic coordinates were optimized in accordance with the forces acting on the atoms (the optimization method is described in [24]). The total energy minimum was considered to have been achieved if the total energy variation around the minimum did not exceed $0.001 \mathrm{eV}$. Thus optimized, the parameters of crystal structure turned out to be rather close to the parameters of undoped anatase. For instance, the calculated lattice parameters for carbon-doped anatase amounted to $a=3.7946 \AA, c / a=2.5295$, and for nitrogen-doped anatase the parameters were obtained at the values of $=3.7958 \AA$ and $c / a=2.5208$, while the corresponding perfect anatase lattice parameters were $3.7845 \AA$ and 2.5140 , respectively. For the optimized geometry, the total densities of states (DOS's) obtained by tetrahedron integration [24] were plotted.

Modeling the vacancies having a charge of $q=+1,+2$ was carried out by introducing one atom of carbon or nitrogen into the supercell. It was demonstrated in [25] that with synthesis under low partial oxygen pressure under thermodynamic equilibrium conditions, the atoms of carbon or nitrogen have a tendency of forming vacancy-dopant pairs. Therefore, in the present study we assumed that the dopants of carbon or nitrogen substitute for the oxygen atoms nearest to vacancy.

Optical absorption coefficients were calculated for all considered variants of the defects in anatase. The calculations were based on the energy-dependent dielectric function that was calculated within the independent particle approximation [24]. The absorption coefficient was calculated from the real and imaginary parts of the dielectric function according to the theory described in [26]. Specifically, within the theory, the absorption coefficient is calculated as follows:

$$
K(E)=\frac{\epsilon_{2}(E)}{2} \frac{1}{\left\{\frac{\epsilon_{1}(E)}{2}+\left[\left(\frac{\epsilon_{1}(E)}{2}\right)^{2}+\left(\frac{\epsilon_{2}(E)}{2}\right)^{2}\right]^{1 / 2}\right\}^{1 / 2}}
$$


where $\epsilon_{1}$ and $\epsilon_{2}$ are, respectively, the excitation energy-dependent real and imaginary parts of the dielectric function averaged over directions of the wave vector.

The electron density functional theory, including the PAW method, is known to have a drawback that the bandgap value in semiconductors is underestimated, which can result in erroneous calculations of optical characteristics. To eliminate such errors, we used a so-called LDA+U approach, within which the exchange-correlation potential is impoved by introducing one-center Hubbard-type corrections. An option suggested by Dudarev et al in [27] was used, where the exchange-correlation corrections depend on one parameter $U$. In all our calculations, we assume the parameter to be $U=7.8 \mathrm{eV}$, which brings the bandgap of the perfect anatase in a good agreement with experimental data, i.e., $3.2 \mathrm{eV}[28]$.

\section{Oxygen vacancy formation energy and the effect of doping with carbon or nitrogen}

When calculating the vacancy formation energy in anatase, the entropy contribution was neglected and only the first-principle calculations of the total energy were taken into consideration. In this approximation, the free energy of oxygen vacancy formation in the 96 -atom anatase cell can be written as follows:

$$
E_{f}(v a c)=E_{t o t}\left(\mathrm{Ti}_{32} \mathrm{O}_{63}\right)+\mu(\mathrm{O})-E_{t o t}\left(\mathrm{Ti}_{32} \mathrm{O}_{64}\right) .
$$

Here, $\mu(\mathrm{O})$ is the chemical potential of oxygen atom. Similarly, if a substitutional dopant for oxygen atom $\mathrm{A}_{\mathrm{O}}$, where A states for $\mathrm{C}$ or $\mathrm{N}$, is added to the cell, then the vacancy formation energy can be calculated as:

$$
E_{f}\left(v a c, \mathrm{~A}_{\mathrm{O}}\right)=E_{t o t}\left(\mathrm{Ti}_{32} \mathrm{O}_{62} \mathrm{~A}_{\mathrm{O}}\right)+\mu(\mathrm{O})-E_{t o t}\left(\mathrm{Ti}_{32} \mathrm{O}_{63} \mathrm{~A}_{\mathrm{O}}\right) .
$$

The method for calculating the chemical potential for oxygen atom was thoroughly described in [25], so here, we only note that this method is based on the calculation of the total energy of oxygen molecule, and includes also the temperature and the partial pressure dependences. Fig. 2 displays the values of chemical potential for three temperatures ranging from room temperature to a temperature of $T=900 \mathrm{~K}$, which is a typical value for annealing during synthesis from precursors. We also assume that the partial oxygen pressure can be varied from 1 to $10^{-30}$ atm (conditional vacuum).
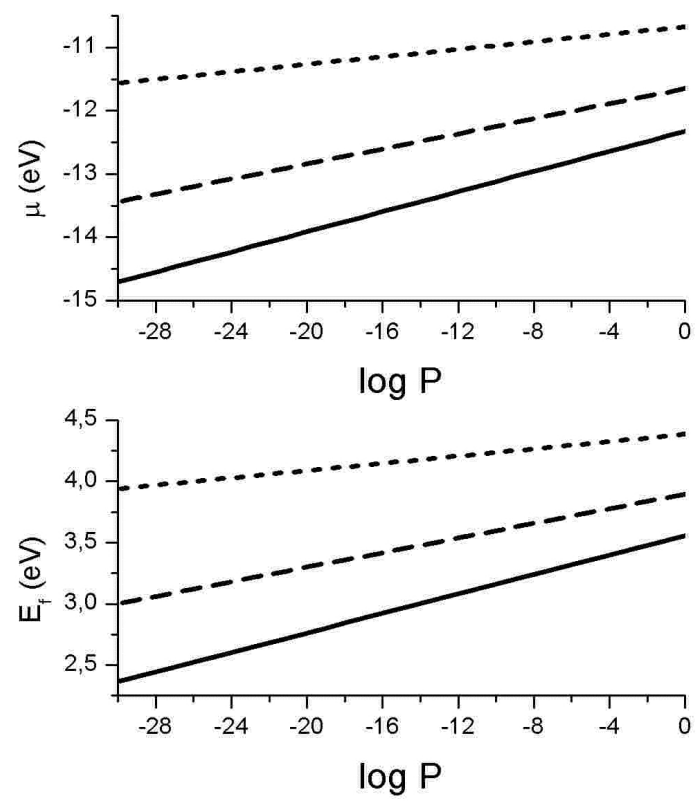

FIG. 2. Calculated values of the chemical potential for oxygen atom (upper panel) and vacancy formation energy (lower panel) in anatase as a function of temperature and partial oxygen pressure $P$. Solid lines designate the results for $T=900$; dashed lines designate the result for 600 , and dotted lines correspond to $T=300$

It can be seen that the chemical potential values and, accordingly, the vacancy formation energy values, decrease as temperature increases and partial pressure decreases. However, at all the considered values of $T$ and $P$, the oxygen vacancy formation energy retains a great positive value, i.e., in undoped anatase under thermodynamic equilibrium 
synthetic conditions of the formation of vacancies at a high concentration is not feasible. This conclusion agrees with the outcomes reported in $[13,14]$, even though the values of vacancy formation energy are somewhat different from the ones reported in our study.

The vacancy formation energy values in the presence of carbon or nitrogen dopants as a function of pressure and temperature are shown in Fig. 3.

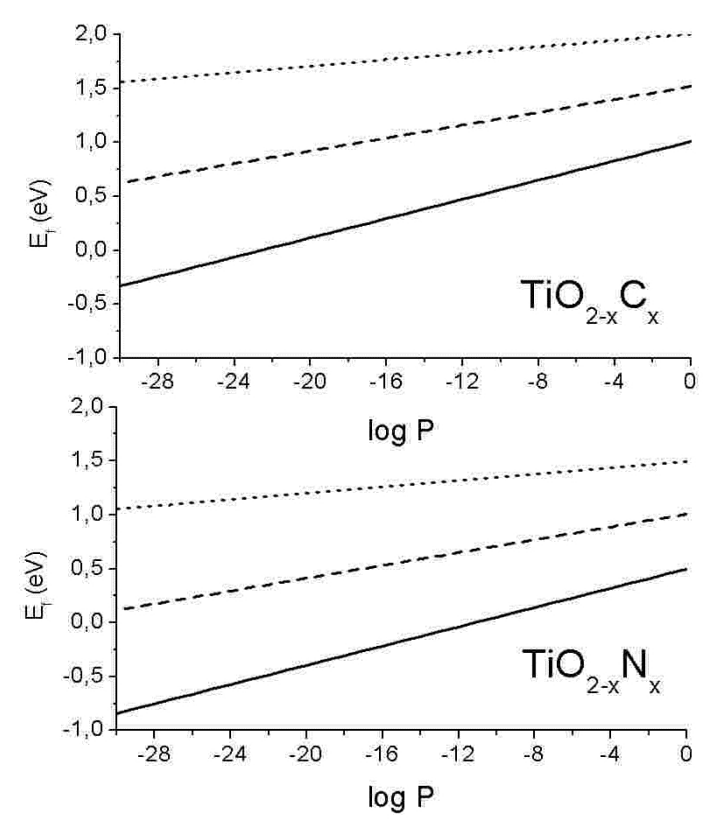

FIG. 3. Calculated values of vacancy formation energy in anatase in the presence of carbon or nitrogen dopants as a function of temperature and partial oxygen pressure $P$. The solid lines indicate the results obtained at a temperature of $T=900$; the dashed line corresponds to $T=600$; and the dotted line corresponds to $T=300$

It can be seen that introduction of carbon or nitrogen atoms into the anatase structure results in a significant decrease in the vacancy formation energy. The energy values become negative at a temperature of 900 and at the partial oxygen pressure $P=10^{-24}$ atm for doping with carbon and at the pressure $P=10^{-12}$ atm for doping with nitrogen, which is indicative of a possibility to significantly increase the vacancy concentration with the presence of these dopants. This outcome is also in agreement with the conclusions reported in [13,14]. It can also be seen that the presence of nitrogen results in a more significant decrease in the vacancy formation energy. However, we will show below that increasing the photocatalytic activity of $\mathrm{N}$-doped anatase is unlikely to be achieved by increasing vacancy concentration in anatase.

\section{Electronic structure, optical absorption and photocatalytic activity of partially reduced anatase in the presence of carbon or nitrogen dopants}

Figure 4 displays densities of states for pristine anatase and for anatase containing 1.56 atom\% of oxygen vacancies. Showing a good agreement with experiment, the bandgap width is $3.21 \mathrm{eV}$ for undoped anatase; and it is $3.12 \mathrm{eV}$ for anatase with vacancies. In the presence of vacancies, below the bottom of conduction band at $5.89 \mathrm{eV}$, there appears a band of vacancy states which contains two electrons and, because of the lack of electron transfer into lower-energy states, is electrically neutral. There is no gap between the vacancy band states and the conduction band states; this agrees with the results reported in [29], according to which the width of bandgap in partially-reduced anatase does not exceed $0.01 \mathrm{eV}$. Therefore, along with the fundamental absorption, one should expect optical absorption occurring over the entire visible range and near IR due to the excitations from the vacancy states into the conduction band. This is supported quite well with the experimental data reported in [30], which are presented in Fig. 5.

Figure 6 displays the densities of states observed with nitrogen or carbon atoms being introduced into partially reduced anatase; and Fig. 7 shows the corresponding data obtained in the calculations of absorption coefficients. The states of nitrogen atom are localized near the bandgap bottom, and the vacancy states are located near the upper edge of the bandgap. If a nitrogen atom substitutes for an oxygen atom, then it traps one extra electron from the vacancy states, which corresponds to the transition of a vacancy into the state with a charge of $q=+1$. It follows from this 

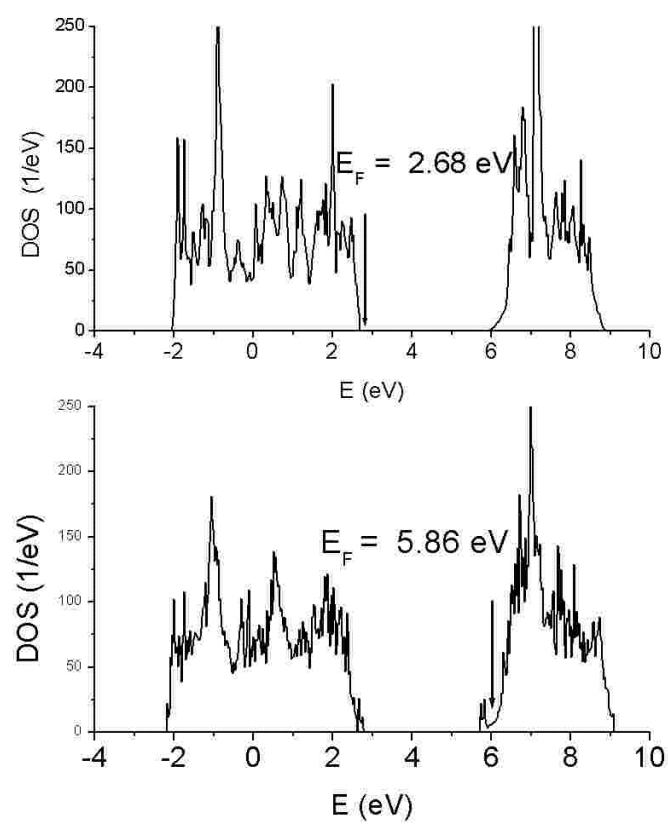

FIG. 4. Density of states in the valence and conduction bands as calculated in the LDA+U approximation for undoped anatase (upper panel) and for anatase containing oxygen vacancies (lower panel). Marked are the Fermi level positions

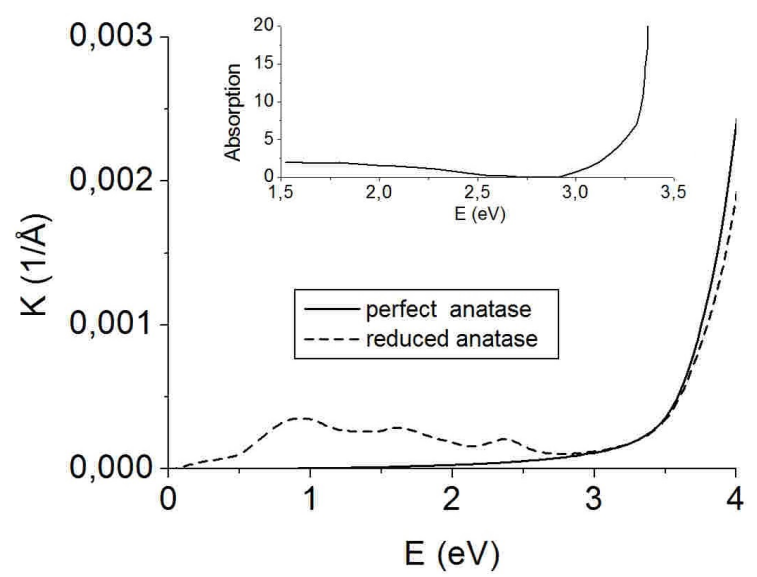

FIG. 5. Calculated absorption coefficients for perfect anatase and that having vacancies in the oxygen sublattice, and the corresponding experimental data reported in [29], in the insert

band structure that, as far as light absorption is concerned, a situation is realized that is similar to the situation for undoped anatase. Really, it can be seen from Fig. 7 that over the entire optical range the absorption profile is rather close to the profile of absorption in the reduced anatase.

However, the situation changes significantly with the presence of carbon atoms. In this case, inside the bandgap there appear two narrow bands of carbon states near 3 and $4 \mathrm{eV}$, with the Fermi level being positioned at the upper edge of the second band. Two electrons from the vacancy states drop to these states, i.e., a vacancy takes the charge $q=+2$. The band near $3 \mathrm{eV}$ is separated from the empty vacancy states with an interval of $2.8 \mathrm{eV}$, and the band near $4 \mathrm{eV}$ is separated with an interval of $1.9 \mathrm{eV}$. It can be seen from Fig. 7 that near $2.8 \mathrm{eV}$, the light absorption is nearly ten times as great as the absorption in reduced undoped anatase. This can lead to increased photocatalytic activity because of the increased number of light quanta absorbed. Comparison with the densities of states demonstrates that the absorption peak at $2.8 \mathrm{eV}$ is partially caused by the transitions from the carbon atomic states near $3 \mathrm{eV}$ into the vacancy states. The second reason for this peak may be the excitations from the carbon band near $4 \mathrm{eV}$ into the states of conduction band that form a peak at $6.8 \mathrm{eV}$. 

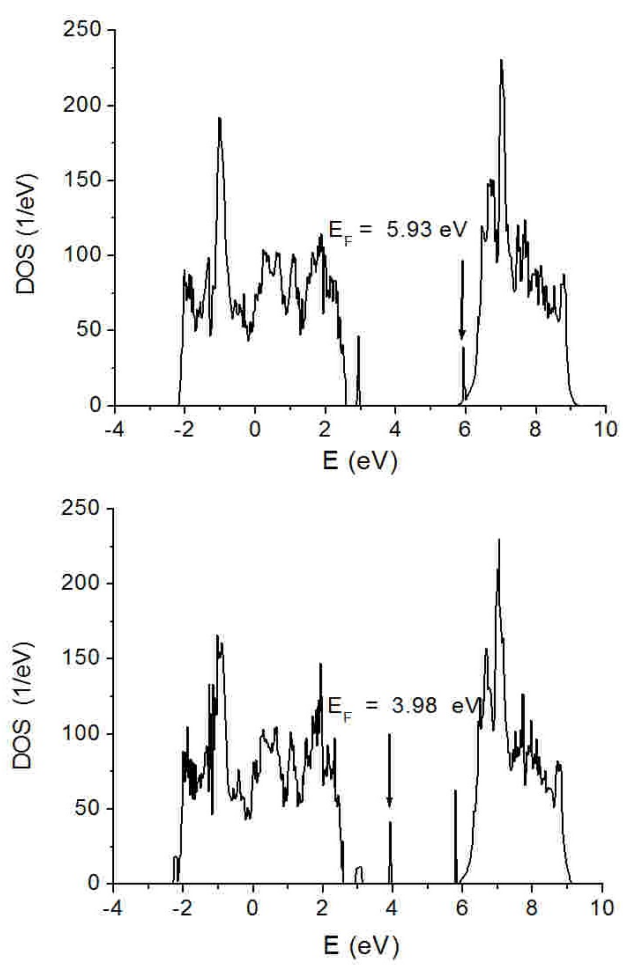

FIG. 6. Density of states in the valence band and in the conduction band, calculated in the LDA+U approximation for anatase containing oxygen vacancies and nitrogen atoms (upper panel) or oxygen vacancies and carbon atoms (lower panel). Marked are the Fermi level positions
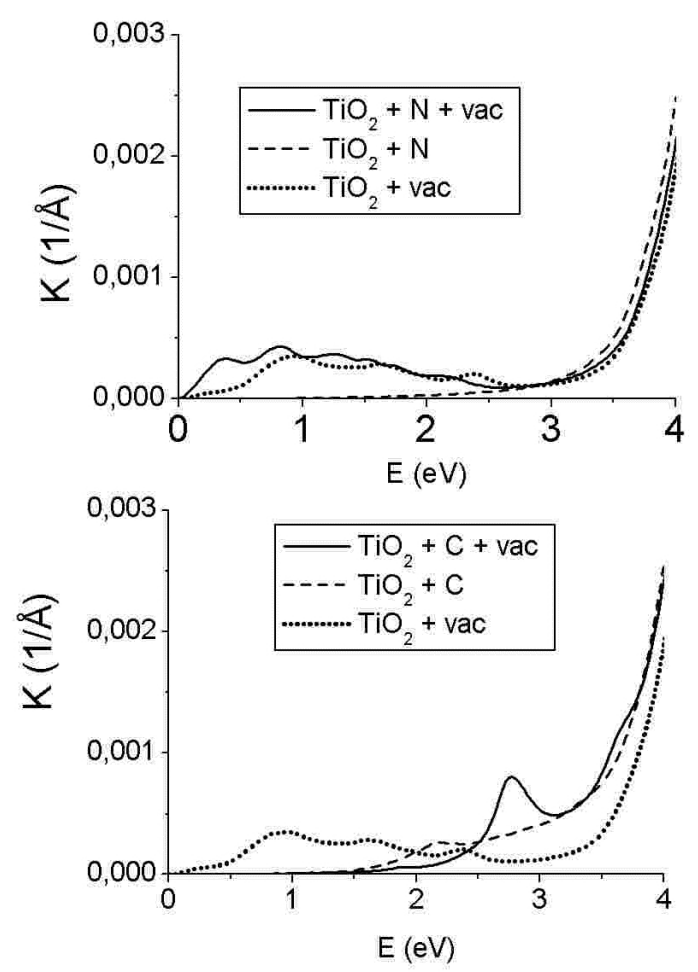

FIG. 7. Calculated values of absorption coefficient of anatase having vacancies in the oxygen sublattice in the presence of nitrogen atoms (upper panel) or carbon atoms (lower panel). Values of absorption coefficients are also given for nitrogen or carbon-doped anatase without oxygen vacancies 


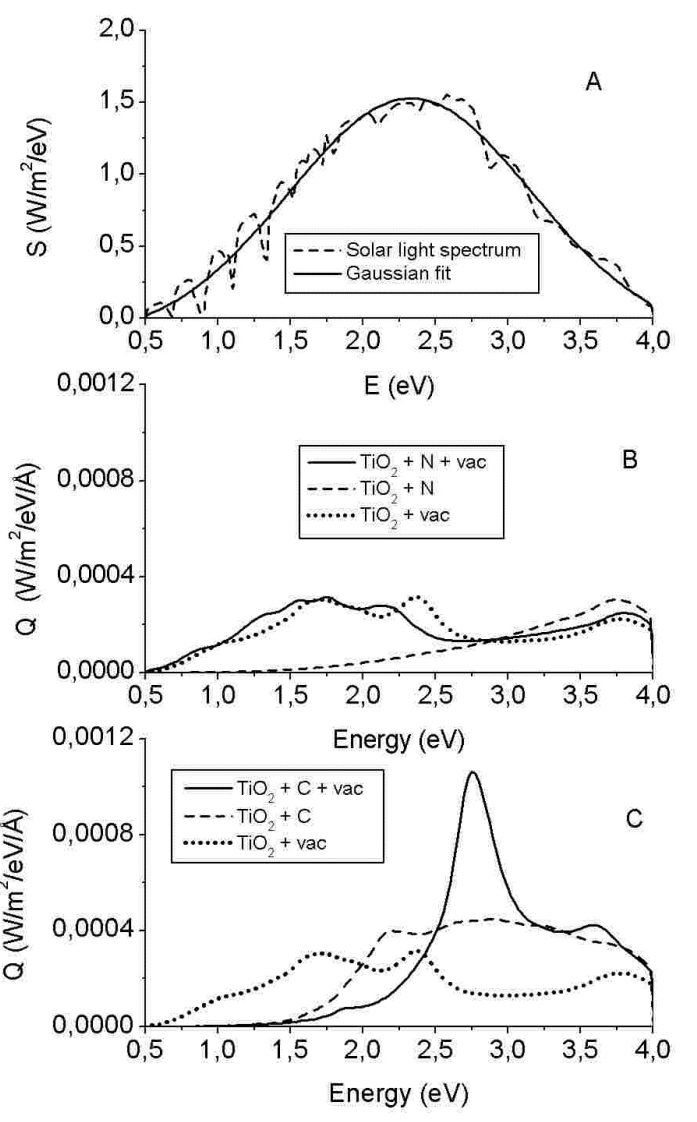

FIG. 8. Panel : solar radiation spectrum [31] and the corresponding Gaussian approximation; panels $\mathrm{B}$ and $\mathrm{C}$ display the calculated values of optical efficiency

It is known that upon passing through the atmosphere the solar radiation spectrum looks like a curve approaching the Gaussian distribution with a width of about $2 \mathrm{eV}$ and a maximum located near $2.5 \mathrm{eV}$. To quantitatively describe the efficiency of solar radiation absorption in the materials under study, we introduce a notion of a catalyst's optical efficiency defined as $Q(E)=S(E) \times K(E)$, where $S(E)$ is the Gaussian approximation to the solar radiation spectrum (see Fig. 8), and also the notion of an integral efficiency defined as $I_{Q}=\int Q(E) d E$, which upon calculation, we confine inside the interval from 0.5 to $4 \mathrm{eV}$. The results for $I_{Q}$ are given in Table 1 .

TABLE 1. Integral values of optical efficiency $I_{Q}$

\begin{tabular}{|c|c|c|c|c|c|c|}
\hline Compound & $\mathrm{TiO}_{2}$ & $\begin{array}{c}\mathrm{TiO}_{2}+ \\
\text { vacancy }\end{array}$ & $\mathrm{TiO}_{2}+\mathrm{N}$ & $\begin{array}{c}\mathrm{TiO}_{2}+\mathrm{N}+ \\
\text { vacancy }\end{array}$ & $\mathrm{TiO}_{2}+\mathrm{C}$ & $\begin{array}{c}\mathrm{TiO}_{2}+\mathrm{C}+ \\
\text { vacancy }\end{array}$ \\
\hline$I_{Q} \times 10^{4}$ & 2.8 & 6.3 & 3.4 & 6.6 & 8.3 & 9.0 \\
\hline
\end{tabular}

Analysis of the data leads to the following conclusions. Perfect anatase, which is seen to absorb only a fraction of UV part of solar radiation, has the lowest optical efficiency, which is nearly one third as much as the optical efficiency of the other materials under study. Since doping with nitrogen causes just a slight red-shift of the fundamental absorption edge, the lack of absorption in the visible spectrum for $\mathrm{TiO}_{2}+\mathrm{N}$ causes only a small increase in the integral quantum efficiency. However, for partially reduced anatase, both in the $\mathrm{TiO}_{2}+$ vacancy and $\mathrm{TiO}_{2}+\mathrm{N}+$ vacancy cases, the absorption in the visible spectrum results in a doubled increase of $I_{Q}$ as compared to the perfect anatase. The maximum integral efficiency is predicted for $C$-doped anatase, as can be seen from the data for $\mathrm{TiO}_{2}+\mathrm{C}$ and $\mathrm{TiO}_{2}+\mathrm{C}+$ vacancy cases. The reason is that these compounds exhibit in creased absorption near the maximum of solar radiation spectrum.

It should be noted, however, that there are certain properties of the band structure, which hinder any significant increase in the photocatalytic activity under doping, regardless of the absorption observed in the visible spectrum and 
despite the increased values of optical efficiency. A necessary condition for photocatalytic activity is a sufficiently long time required for the recombination of electron-hole pairs, which should be greater than the time for transferring excited carriers into the reaction medium. It is known that the picoseconds or greater time is required for the recombination of pairs formed via the interband excitations in semiconducting oxides [32,33]. It is also known that a time of no less than ten ps is required for transferring electrons onto the islands of a sensibilizator (e.g. the time for transferring electrons from $\mathrm{TiO}_{2}$ onto platinum islands on the surface), and even a greater time (up to $100 \mathrm{~ms}$ ) is required, for example, for transferring electrons on oxygen molecules on the surface, see a review reported in [33]. However, one should expect that the recombination time is going to be several orders of magnitude smaller if there is no gap between the vacancy states and the conduction band, as is the cases for $\mathrm{TiO}_{2}+$ vacancy and $\mathrm{TiO}_{2}+\mathrm{N}+$ vacancy. It was shown in [34] that, because of the emission of phonons, the excited electrons in the conduction band states of $\mathrm{TiO}_{2}$ lose their excitation energy and sink onto the bottom of the band over the time that does not exceed several tens of fs; after this time the electrons recombine with the holes in the vacancy states. These data are also in agreement with the experimental results which reveal that the trapping of conduction band electron onto the states of dopants near the top of the band gap occurs over $100-250 \mathrm{fs}$, while the hole trapping on the states near the bottom of the gap takes $50-150 \mathrm{fs}$ [33]. Therefore, one can expect that for these cases, only a small number of electrons are involved in the photocatalytic process. These are the excited electrons which form a high-energy "tail" of the electron distribution in the conduction band [34]; in analogy, a similar distribution of holes inside the valence band can exist [35]. In both cases, as in $\mathrm{TiO}_{2}+$ vacancy as in $\mathrm{TiO}_{2}+\mathrm{N}+$ vacancy, the electron-hole pairs can be formed not only because of the excitations inside the conduction band but also because of the excitations into the conduction band from the valence band or from the band of nitrogen states. However, the energy of such excitations is greater than $3 \mathrm{eV}$, which also prevents any significant increase in the photocatalytic activity as compared to the perfect anatase. It should be noted that, although the nitrogen-doped anatase is a classical subject of photocatalytic studies, there are data available that report about its rather small photocatalytic activity. Thus, in [36-39], the photocatalytic activity of N-doped anatase has been studied in the decomposition reactions of formic acid, ethylene glycol and other compounds, but no positive effect of $\mathrm{N}$-doping has been revealed

Therefore, only in the $\mathrm{TiO}_{2}+\mathrm{C}+$ vacancy case, that is, for the $\mathrm{C}$-doped partially-reduced anatase, we can expect an increase in the photocatalytic activity caused by the optical absorption accompanied by the long time required for the recombination of excited electron-hole pairs. To our knowledge, there are no systematic data available on the photocatalytic activity of similar compounds; however,the photocatalytic activity of C-doped anatase observed in the visible spectrum has repeatedly been reported in literature (see, for instance, the literature review in [36]). Our calculations provide evidence that the photocatalytic activity of $\mathrm{C}$-doped anatase can be increased through its partial reduction.

\section{Conclusion}

Based on the first-principle calculations of the electronic band structure and oxygen vacancy formation energy for undoped anatase and that containing carbon or nitrogen dopants, we analyzed the influence of vacancies on the photocatalytic activity in visible spectrum. It was demonstrated for undoped anatase that a high vacancy concentration cannot be obtained via synthes is under thermodynamic equilibrium conditions, neither at an elevated temperature nor at a low partial oxygen pressure. In addition, because of the short electron-hole recombination time and because of the high fundamental absorption energy, no significant increase in the photocatalytic activity can be observed for reduced undoped anatase.

We also demonstrate that the vacancy formation energy can be decreased considerably, i.e., the vacancy concentration can be increased, in the presence of carbon or nitrogen atoms substituting the oxygen atoms. This effect is especially pronounced for nitrogen dopants. However, with nitrogen doping, because of the excessive energy of fundamental absorption and short time of electron-hole recombination, one can hardly expect an essential increase in the photocatalytic activity. Our calculations provide evidence that the optical absorptionmay increase in partially reduced $\mathrm{C}$-doped anatase because of the interband transitions at the energy approaching the solar radiation intensity maximum. This might be a result of tuning the electronic band structure via creation of new states near the middle of the band gap and beneath the bottom of the conduction band.

\section{Acknowledgements}

The calculations were carried out on the URAN cluster of the Institute of Mathematics and Mechanics, Ural Branch of the Russian Academy of Sciences. A deep gratitude is expressed to V. N. Krasilnikov for the fruitful discussions of the research subject. 


\section{References}

[1] Hoffmann M.R., Martin S.T., Choi W., Bahnemann D.W. Environmental applications of the semiconductor photocatalysis. Chem. Rev., 1995, 95 (1), P. 69-96.

[2] Fujishima A., Rao T.N., Tryk D.A. Titanium dioxide photocatalysis. J. Photochem. Photobiol. C: Photochem. Rev., 2000, 1 (1), P. 1-21.

[3] Hashimoto K., Irie H., Fugishima A. $\mathrm{TiO}_{2}$ photocatalysis: a hystorical overview and future prospects. Jap. Journ. Appl. Phys., 2005, 44 (12), P. 8269-8285.

[4] Zaleska A. Doped-TiO 2 : a review. Recent Patents on Engineering, 2008, 2 (3), P. 157-164.

[5] Kitano M., Tsujimaru M., Anpo M. Hydrogen producing using highly active titanium oxide-based photocatalysts. Top. Catal., 2008, 49 (1-2), P. 4-17.

[6] Thakur R.S., Chaudhary R., Singh C.J. Fundamentals and applications of the photocatalytic treatment for the removal of industrial organic pollutants and effects of operational parameters: a review. Renewable Sustainable Energy, 2010, 2 (4), P. 042701-04399.

[7] Gupta S.M., Tripathi M. A review of $\mathrm{TiO}_{2}$ nanoparticles. Chinese Sci. Bull., 2011, 56 (16), P. 1639-1657.

[8] Justicia I., Ordejón P., et al. Designed self-doped titanium oxide thin films for efficient visible-light photocatalysis. Advanced materials, 2002, 14 (19), P. 1399-1402.

[9] Martyanov I.N., Uma S., Rodrigues Sh., Klabunde K.J. Structural defects cause $\mathrm{TiO}_{2}$-based photocatalysts to be active in visible light. Chem. Commun., 2004, 21, P. 2476-2477.

[10] Liu H., Yang W., Ma Y., Yao J. Extended visible light response of binary $\mathrm{TiO}_{2}-\mathrm{Ti}_{2} \mathrm{O}_{3}$ photocatalist prepared by a photo-assisted sol-gel method. Applied Catalysis A, 2006, 299 (1), P. 218-223.

[11] Dong C.X., Xian A.P., Han E.H., Shang J.K. Acid-mediated sol-gel synthesis of visible-light active photocatalists. Journ. of Mater Sci., 2006, 41 (18), P. 6168-6170.

[12] Yang X., Cao Ch., et al. Synthesis of visible-light-active $\mathrm{TiO}_{2}$-based photocatalysts by carbon and nitrogen doping. Journ. of Catalysis, 2008, 260 (1), P. 128-133.

[13] Di Valentin C., Pacchioni G., Selloni A. Theory of carbon doping of titanium dioxide. Chem. Mater., 2005 , 17 (26), P. 6656-6665.

[14] Di Valentin C., Finazzi E., et al. N-doped $\mathrm{TiO}_{2}$ : theory and experiment. Chem. Phys., 2007, 339 (1-3), P. 44-56.

[15] He J., Sinnot S.B. Ab initio calculations of intrinsic defects in rutile TiO $\mathrm{T}_{2}$. Journ. Amer. Ceram. Soc., $2005, \mathbf{8 8}$ (3), P. 737-741.

[16] He J., Behera R.K., et al. Prediction of high-temperature point defect formation in $\mathrm{TiO}_{2}$ from combined ab initio and thermodynamic calculations. Acta Materialia, 2007, 55 (13), P. 4325-4337.

[17] Xiao Q., Si Zh., et al. Effect of samarium dopant on photocatalytic activity of $\mathrm{TiO}_{2}$ nanocrystallite for methylen blue degradation. Journ. of Mater. Sci., 2007, 42 (22), P. 9194-9199.

[18] Islam M.M., Bredow T., Gerson A. Electronic properties of oxygen-deficient and aluminium-doped rutile TiO 2 from first principles. Phys. Rev. B, 2007, 76 (4), 045217.

[19] Osorio-Guillén J., Lany S., Zunger A. Atomic control of conductivity versus ferromagnetism in wide-gap oxides via selective doping: V, Nb, Ta in anatase $\mathrm{TiO}_{2}$. Phys. Rev. Lett., 2008, 100 (3), 036601.

[20] Zainullina V.M., Zhukov V.P., et al. Electronic structure and the optical and photocatalytic properties of anatase doped with vanadium and cabon. Physics of the Solid State, 2010, 52 (2), P. 271-280.

[21] He J., Finnis M.W., Dickey E.C., Sinnott S.B. Charged defects formation energies in $\mathrm{TiO}_{2}$ using the supercell approximation. Advances in Science and Technology, 2006, 45, P. 1-8.

[22] Payne M.C., Teter M.P., et al. Iterative minimization techniques for ab initio total-energy calculations: molecular dynamics and conjugate gradients. Rev. of Modern Physics, 1992, 64 (4), P. 1045-1098.

[23] Diebold U. The surface science of titanium dioxide. Surface Science Reports, 2003, 48 (5-8), P. 53-229.

[24] Kresse G., Marsman M., Furthmuller J. Vasp the guide. 2011. URL: http: / / cms . mpi . univie.ac.at/vasp/guide/vasp.html.

[25] Zhukov V.P., Shein I.R. Ab initio thermodynamic characteristics of the formation of oxygen vacancies, and boron, carbon, and nitrogen impurity centers in anatase. Physics of the Solid State, 2018, 60 (1), P. 37-48.

[26] Ziman J.M. Principles of the Theory of Solids. Cambridge University Press, 2013, 452 p.

[27] Dudarev S.L., Botton G.A., et al. Electron-energy loss spectra and structural stability of nickel oxide: an LSDA+U study. Phys. Rev. B, 1998, 57 (3), P. 1505-1509.

[28] Tang H., Berger H., et al. Photoluminescence in $\mathrm{TiO}_{2}$ anatase single crystals. Solid State Commun., 1993, 87 (9), P. 847-850.

[29] Jacímovic J., Vaju C., et al. Pressure dependence of the large-polaron transport in anatase. EPL, 2012,99 (5), 57005.

[30] Sekiya T., Yagisawa T., et al. Defects in anatase $\mathrm{TiO}_{2}$ single crystal controlled by heat treatment. Journ. of the Phys. Soc. of Japan, 2004, 73 (3), P. 703-710.

[31] Wu J., Walukiewicz W., et al. Unusual properties of the fundamental band gap of InN. Appl. Phys Lett., 2002 , 80 (21), P. $3967-3969$.

[32] Henderson M.A. A surface science perspective on $\mathrm{TiO}_{2}$ photocatalysis. Surface Science Reports, 2011,66 (6-7), P. 185-297.

[33] Schneider J., Matsuoka M., et al. Understanding $\mathrm{TiO}_{2}$ photocatalysis: mechanisms and materials. Chemical Review, 2014, 114 (19), P. 99199986.

[34] Tyuterev V.G., Zhukov V.P., Chulkov E.V., Echenique P.M. Relaxation of highly excited carriers in wide-gap semiconductors. Journ. of Phys.: Cond. Matter, 2015, 2, 02581.

[35] Zhukov V.P., Tyutetev V.G., Chulkov E.V., Echenique P.M. Hole-phonon relaxation and photocatalytic properties of titaniu dioxide and zinc oxide: first-principle approach. Intern. Journ. of Photoenergy, 2014, Article Id. 738921.

[36] Mrowetz M., Balcerski W., Colussi A.J., Hoffmann M.R. Oxidative power of nitrogen-doped $\mathrm{TiO}_{2}$ photocatalysts under visible illumination. Journ. of Phys. Chem. B, 2004, 108 (45), P. 17269-17273.

[37] Irie H., Watanabe Y., Hashimoto K. Nitrogen-concentration dependence on photocatalytic activity of TiO ${ }_{2-x} \mathrm{~N}_{x}$ powders. Journ. of Phys. Chem. B, 2003, 107 (23), P. 5483-5486.

[38] Fu H.B., Zhang L.W., et al. Electron spin resonance spin-trapping of radical intermediates in $\mathrm{N}_{\text {-doped }} \mathrm{TiO}_{2}$-assisted photodegradation of 4-chlorophenol. Journal of Phys. Chem. B, 2006, 110 (7), P. 3061-3065.

[39] Tachikawa T., Fujitsuka M., Majima T. Mechanistic insight into the $\mathrm{TiO}_{2}$ photocatalytic reactons: design of new photocatalysts. Journal of Physical Chemistry C, 2007, 111 (14), P. 5259-5275. 